\title{
Formulation of Catechin Hydrate Nanocapsule and Study of its Bioavailability
}

\author{
Arpita Samanta $^{1 *}$, Bidyut Bandyopadhyay ${ }^{1}$ and Nirmalendu Das ${ }^{2}$ \\ ${ }^{1}$ Department of Biotechnology, Oriental Institute of Science and Technology, Vidyasagar University, Dewandighi, Burdwan, West Bengal, India \\ ${ }^{2}$ Drug Development Diagnostics and Biotechnology, CSIR - Indian Institute of Chemical Biology, Jadavpur, Kolkata, West Bengal, India
}

\begin{abstract}
Flavonoid is a polyphenolic antioxidant used to treat free radical mediated tissue damage. So flavonoids are the good targets for treatment of many diseases like cancer, toxicity health hazards, neurodegenaration etc. Drug delivery of flavonoid is the novel approach where dosing and drug toxicity can be minimized. Antioxidant Catechin Hydrate $(\mathrm{CH})$ is choosen to make drug delivery vehicle: nanocapsules either alone or in combination with chelator sodium meta borate (SMB) within biodegradable poly-lactide-co-glycolide (PLGA). Sizes of the prepared particles were determined through scanning and atomic force microscopy where less than $50 \mathrm{~nm}$ particle sizes were revealed. Bioavailability in serum study determines that shelf life of $\mathrm{CH}$ can be increased with nanocapsule delivery compared to free drug. Results demonstrate the efficacy of Nanocapsulated $\mathrm{CH}$ to be a potent vehicle for tissue targeting.
\end{abstract}

Keywords: Drug delivery; Nanocapsule; Flavonoid; Catechin hydrate; Sodium meta borate; Bioavailability

Abbreviations: NaF: Sodium Fluoride; $\mathrm{CH}$ : Catechin Hydrate; SMB: Sodium Meta Borate; NP: Nano Particle; NPCH: Nano Particulate Catechin Hydrate; NPSMB: Nano Particulate Sodium Meta Borate; NP (CH+SMB): Nano Particle Encapsulated With Catechin Hydrate and Sodium Meta Borate; PLGA: Poly Lactide-Co-Glycolide; DMAB: Didodecyldimethylammonium Bromide; BBB: Blood Brain Barrier; HPLC: High Pressure Liquid Chromatography; SEM: Scanning Electron Microscopy; AFM: Atomic Force Microscopy; DLS: Dynamic Light Scattering; PBS: Phosphate Buffered Saline.

\section{Introduction}

Drug delivery refers to approaches, formulations, technologies, and systems for transporting a pharmaceutical compound in the body as needed to safely achieve its desired therapeutic effect. It may involve scientific site targeting within the body, and also facilitating systemic pharmacokinetics; in any case, it is typically concerned with both quantity and duration of drug's persistence in the system. Drug delivery is often executed via a drug's chemical formulation alone, but it may also involve in drug-device combination products. Drug delivery is a concept where much emphasis should be given to dosage form and route of administration.

The choice of the delivery systems is to allow the safe application of the drug with minimum losses. This suggests that the drug in the formulation must be chemically, physically and microbiologically stable. Side-effects of the drug and drug interactions should be avoided or minimized by the use of suitable drug delivery systems.

In this study, poly lactic-co-glycolic acid (PLGA) was used as a carrier polymer for nano encapsulation of Catechin Hydrate $(\mathrm{CH})$, an herbal polyphenolic flavonoid. Flavonoids have limited water solubility even if they are in the form of glycosides, thus showing poor bioavailability, which can be easily modified by environmental factors such as temperature, $\mathrm{pH}$ and light. The mechanisms of gastrointestinal absorption of flavonoids are complex which are poorly absorbed in their natural form in the intestine. So flavonoids result in poor bioavailability, poor permeability, instability and extensive first-pass bioavailability of flavonoids, such as low water solubility, instability to gastric and colonic $\mathrm{pH}$, metabolism by gut microflora, and absorption across the intestinal wall, active efflux mechanism and first pass metabolic effects. Nano carriers could be utilized to enhance bioavailability and bioefficacy of flavonoids because they can increase solubilization potential, alter absorption pathways, and prevent the metabolic degradation within the gastrointestinal tract [1] Catechins, especially those present in green tea, have received considerable attention in recent days due to their favourable biological properties but their therapeutic potential is limited by their low oral bioavailability $(<5 \%)$ attributed to poor stability and intestinal absorption and a short half-life owing to strong systemic clearance [2]. The present study hypothesized that nano encapsulation of Catechin hydrate $(\mathrm{CH})$ with PLGA will improve the $\mathrm{CH}$ stability by protecting capsule from oxidation and improve its aqueous solubility, biological availability, and bioactivity.

Cationic boranes form chelate complexes with fluoride anions [3]. Catechin hydrate, a polyphenolic flavonol antioxidant exhibits its properties against many diseases including ischemic neurodegeneration, atherosclerosis, stomach cancer etc. [4-6]. Catechin Hydrate $(\mathrm{CH})$, a secondary metabolite from green tea, has been associated with antioxidant, anti-inflammatory, anti-proliferative, anticancer, anti-angiogenic, and anti-diabetic activities. However poor water solubility and limited oral bioavailability are the major roadblocks in its development as a therapeutic agent for cancer and other chronic diseases. Previous report has already established the potency of combined therapy by chelator and antioxidant drug to prevent arsenic induced chronic toxicity in rats [7]. Hence an improved combinatorial delivery mode for (+)-Catechin hydrate and sodium metaborate could be expected as an effective approach to reduce fluoride induced oxidative stress.

The use of nanoparticles as drug carriers has an obvious advantage over existing approaches to circumvent the blood brain barrier. Loaded with both hydrophobic and hydrophilic drugs, this colloidal nanoparticles drug carrier has the ability to cross both the GI tract

${ }^{*}$ Corresponding author: Arpita Samanta, Department of Biotechnology, Orienta Institute of Science and Technology, Vidyasagar University, Dewandighi, Burdwan-02, West Bengal, India, Tel: +913422622175; E-mail: arpita.pari@gmail.com

Received May 30, 2016; Accepted June 14, 2016; Published June 20, 2016

Citation: Samanta A, Bandyopadhyay B, Das N (2016) Formulation of Catechin Hydrate Nanocapsule and Study of its Bioavailability. Med chem (Los Angeles) 6 : 399-404. doi:10.4172/2161-0444.1000376

Copyright: ( $) 2016$ Samanta A, et al. This is an open-access article distributed under the terms of the Creative Commons Attribution License, which permits unrestricted use, distribution, and reproduction in any medium, provided the original author and source are credited. 
mucosal barrier and the blood brain barrier (BBB) to enhance drug bioavailability via particle uptake mechanisms and therefore, it appears to have significant promise in delivering therapeutic molecules [8].

Hence, the aim of the study was to synthesize biodegradable $\mathrm{CH}$ PLGA nanocapsules with better hydrophilicity, bioavailability property for the therapeutic delivery. To achieve this objective, scanning electron microscopy (SEM), atomic force microscopy (AFM), and dynamic light scattering (DLS) were used for the characterization of nanocapsules alone or in combined form.

It is also necessary to investigate the scaling up of the developed $\mathrm{CH}-\mathrm{PLGA}$ nanocapsules for better availability in therapeutic uses.

\section{Materials and Methods}

\section{Chemicals}

Poly (lactide-co-glycolide) (PLGA) (Resomer RG 50:50H) and didodecyldimethylammonium bromide (DMAB) were purchased from Aldrich (St. Louis, MO, USA) and Sigma (St Louis, MO, USA), respectively. Ethyl acetate (AR Grade) was purchased from Rankem Fine Chemicals (New Delhi, India). Sodium fluoride (Merck, Darmstadt, Germany) was used for experimental purposes. Fast Green FCF and Sirius Rose BB were acquired from Fluka. Chloroform and methanol were purchased from E. Merck. (+)-Catechin Hydrate was purchased from Sigma Chemicals (St. Louis, MO, USA). Reagents were of analytical grade.

\section{Methods}

Formulation of Catechin Hydrate $(\mathrm{CH})$ nanocapsules: Polylactide coglycolide (PLGA) (50:50 polymer) was dissolved in $1 \mathrm{~mL}$ of ethyl acetate. $5 \mathrm{mg}$ of catechin hydrate was also dissolved in $5 \mathrm{~mL}$ ethyl acetate. Stabilizing agent didodecyl dimethyl ammonium bromide (DMAB) was dissolved in $1 \mathrm{~mL}$ water. Non-ionic detergent (Tween 20), DMAB and PLGA solution was mixed and placed in a rotating magnetic stirrer. Catechin hydrate mixture was added slowly over the mixture drop wise with a constant stirring speed. Precaution should be measured if clots will form. The occurrence of aggregation was checked for regular interval while adding further drops of catechin hydrate. The mixture will rotate for 3 hours for complete removal of organic solvents and gaseous nitrogen is used to remove traces of organic solvents. The suspension was ultra-centrifuged at 105,000 $\times \mathrm{g}$ in Sorval RC 5B Plus using the rotor Sorval T-865 for $1 \mathrm{~h}$. The pellet of nanocapsules was washed with PBS and re suspended in $2 \mathrm{~mL}$ PBS $(0.025 \mathrm{M})$. For future use the pellet can be lyophilised and stored at $-20^{\circ} \mathrm{C}$ temperature.

Study of in vitro drug $(\mathrm{CH})$ release: $\mathrm{CH}$ - PLGA nanocapsules were suspended in $0.025 \mathrm{M}$ phosphate buffer in a glass bottle and placed in a stirring bath rotated at $50 \mathrm{rpm}$ speed at room temperature. Sample $(5 \mathrm{~mL})$ was removed at selected time interval and centrifuge at 25,000 rpm for half an hour. Supernatant was removed and optical density was measured at $280 \mathrm{~nm}$.

Preparation of CH and SMB nanocapsules: A modified emulsiondiffusion-evaporation method [9] was used to make nanocapsules of catechin hydrate and sodium metaborate. $\mathrm{CH}(5 \mathrm{mg})$ was dissolved in $1 \mathrm{~mL}$ ethyl acetate and $5 \mathrm{mg} \mathrm{SMB}$ in $1 \mathrm{~mL}$ water. In brief, $70 \mathrm{mg}$ of PLGA was dissolved in $5 \mathrm{~mL}$ of ethyl acetate at room temperature. The organic solution of PLGA and drug or PLGA and SMB in ethyl acetate was then separately emulsified with $5 \mathrm{~mL}$ of an aqueous phase containing $50 \mathrm{mg}$ of $\mathrm{DMAB}$. The resulting organic/water emulsion was stirred at room temperature before being homogenized at 15,000 rpm for 5 min with a high-speed homogenizer (Polytron PT4000; Polytron Kinematica, Lucerne, Switzerland). The organic solvent was removed by constant stirring on a water bath set at $40^{\circ} \mathrm{C}$. Nitrogen gas was used to remove further traces of organic solvent. The suspension was ultracentrifuged at $35000 \mathrm{rpm}$ in a Sorval RC 5B Plus using the Sorval T-865 rotor for $1 \mathrm{~h}$. The pellet of nanocapsules was washed with phosphate buffered saline (PBS) twice and suspended in 0.25 M PBS.

Characterization of nanocapsule [NPCH and $\mathrm{NP}(\mathrm{CH}+\mathrm{SMB})]$ using atomic force microscopy and scanning electron microscopy: Sample $(5 \mu \mathrm{L}$ ) was deposited onto Ruby mica sheet (ASTM V1 Grade Ruby Mica from MICAFAB, Chennai) for 15-30 minutes. Samples were gently washed with $0.5 \mathrm{~mL}$ of MilliQ water to remove the molecules that was not firmly attached to the mica. Mica sheets are basically negatively charged so molecule binds strongly on the mica surface, after drying the sample by using vacuum dryer. AAC mode AFM was performed using a Pico plus 5500 ILM AFM (Agilent Technologies USA) with a piezoscanner with $9 \mu \mathrm{m}$ maximum range. All images were obtained in aquatic mode with $225 \mu \mathrm{m}$ length cantilever used from nanosensors, USA, having 150-300 KHz resonance frequency. Images were processed by flatten using Pico view1.1 version software (Agilent Technologies, USA). Imaging (3D) has been done through Pico Image Advanced version software (Agilent Technologies, USA).

For SEM, lyophilised samples were coated with a thin layer of carbon, for high resolution electron imaging applications. High energy beam of electrons systematically scans across a surface of samples to reveal signal that gives information about sample topography, morphology by SEM.

Stability of $\mathrm{CH}$ nanocapsules [NPCH and NP $(\mathrm{CH}+\mathrm{SMB})]$ by Dynamic Light Scattering (DLS): The stability of nanocapsules were tested through dynamic light scattering using a mean laser wavelength of $632.2 \mathrm{~nm}$ and a $90^{\circ}$-angle measurement Nanosizer 90ZS (Malvern Instruments, Southborough, MA, USA). The lyophilized powder was dissolved in aqueous buffer and filtered with $0.2 \mathrm{~mm}$ pore sized membrane and then DLS measurements were done to obtain zeta potential.

Bioavailability of NPCH and NP $(\mathrm{CH}+\mathrm{SMB})$ nanoparticles: The mice were divided into three groups ( 2 mice in each group), group 1 received $\mathrm{CH}$ and group 2 received catechin hydrate-loaded nanoparticles $[\mathrm{NPCH}]$ and group 3 received catechin hydrate-sodium meta borate loaded nanoparticles [NP $(\mathrm{CH}+\mathrm{SMB})]$ Catechin hydrate or catechin hydrate nanoparticles were given intravenously $(2.5 \mathrm{mg} /$ $\mathrm{kg}$ ) to each mouse, the blood was collected at different time intervals, serum was separated, and the concentration was determined by HPLC analysis.

\section{Results and Discussion}

Cationic borane is known as a potent chelator for fluoride [3]. It is now important to explore the therapeutic role of boranes and antioxidant catechin hydrate either alone or in combination; delivered through nanocapsule to treat fluoride induced oxidative damage in rats. Further it demonstrates the role of oral application of chelator sodium meta borate and antioxidant catechin hydrate in a nanocapsulated form to prevent the toxic effect of fluoride in response to chronic fluoride exposure.

\section{NPCH and NPSMB nanocapsule characterization}

Successful nanocapsule mediated drug delivery depends upon its size and physiological properties of nanocapsules. Nanocapsules were obtained in range of 9-36 $\mathrm{nm}$ in size (Table 1). The AFM images revealed that nanocapsules have a more or less spherical shape with narrow size distribution. Encapsulation of $\mathrm{CH}$ and $\mathrm{SMB}$ in their nanocapsules were $65 \%$ and $57 \%$, respectively, whereas that of combined $\mathrm{CH}$ and 


\begin{tabular}{|c|c|c|c|}
\hline Sample Type & $\begin{array}{c}\text { Encapsulation } \\
(\%)\end{array}$ & $\begin{array}{c}\text { Mean particle } \\
\text { size }(\mathbf{n m})\end{array}$ & Zeta Potential (mV) \\
\hline $\begin{array}{c}\text { Blank PLGA } \\
\text { Nanocapsules }\end{array}$ & - & $9 \pm 1.13$ & $-10.56 \pm 0.17$ \\
\hline $\begin{array}{c}\text { PLGA-Catechinhydrate } \\
\text { Nanocapsules }\end{array}$ & $65 \pm 2.1$ & $13.25 \pm 2.4$ & $-17.63 \pm 1.32$ \\
\hline $\begin{array}{c}\text { PLGA-SMB } \\
\text { Nanocapsules }\end{array}$ & $57 \pm 4.8$ & $23.31 \pm 3.1$ & $-15.45 \pm 0.18$ \\
\hline $\begin{array}{c}\text { Co-encapsulated PLGA- } \\
\text { Catechin hydrate: } \\
\text { SMB Nanocapsules }\end{array}$ & $61 \pm 5.7$ & $36.33 \pm 0.67$ & $-19.08 \pm 1.24$ \\
\hline
\end{tabular}

Results were expressed as mean \pm SD of 6 replica for each formulation

Table 1: Nanocapsules composition, percentage encapsulation, size and stability.

SMB was $61 \%$ (Table 1). Encapsulation of drug increases the size of nanocapsules. Percentage of encapsulation signifies the amount of drug loaded in the nanocapsule, which ultimately affect its potency during delivery. Both particles showed good stability in term of negative zeta potential (Figure 1). Negative zeta potential of all prepared nanocapsule proves its stability below $-25 \mathrm{mV}$. Following the initial heavy release sustained drug release profile was shown after 70 hours (Figure 2). The topography-flatten, amplitude and phase-flatten view were observed in Figure 3 with size of $36 \mathrm{~nm}$. Size difference and dimension of free nanoparticles were compared with three drug loaded particle, viz $\mathrm{NPCH}, \mathrm{NPSMB}$ and $\mathrm{NP}(\mathrm{CH}+\mathrm{SMB})$ under $\mathrm{AFM}$ and maximum size was observed in $\mathrm{NP}(\mathrm{CH}+\mathrm{SMB})$ (Figure 3). Smaller nanoparticles less than $50 \mathrm{~nm}$ were observed in our study under AFM (Figures 3 and 5) with no aggregation. The blank nanocapsules had the smallest mean size and the NP $(\mathrm{CH}+\mathrm{SMB})$ nanocapsules showed the largest size among the four types of particles studied under AFM (Figure 4). Stable drug release and drug uptake depend upon the size and distribution of particles [10]. The relationship of particle size was $\mathrm{NPCH}<\mathrm{NPSMB}<\mathrm{NP}$ $(\mathrm{CH}+\mathrm{SMB})$. Three dimensional image of nanoparticle showed its height of $6.95 \mathrm{~nm}$ of $\mathrm{NP}(\mathrm{CH}+\mathrm{SMB})$ on mica sheet (Figure 5). The Scanning electron microscopy (Figure 6) demonstrated the sizes of the prepared nanoparticles were more or less uniform and appropriate for oral administration to rats. Stability of nanocapsules was studied for empty and drug loaded capsules. Size range, aggregation have been evaluated under atomic force microscope (Figures 3-5) and scanning electron microscope (Figure 6) and it indicates particle size below 100 $\mathrm{nm}$, which makes not only facilitated delivery in target organs like liver and kidney but also to brain, which is the ultimate further challenge to cross the BBB. BBB can be crossed effectively by particles lesser than 50 $\mathrm{nm}$ in size during drug targeting to brain cells [11].

\section{Bioavailability of NPCH and NPSMB}

One of the major reasons for making nanocapsulated Catechin hydrate $[\mathrm{NPCH}]$ and combined catechin hydrate-sodium meta borate [NP $(\mathrm{CH}+\mathrm{SMB})]$ are to examine its effect on bioavailability in vivo. For this, mice were intravenously administered either $\mathrm{CH}$ (free) or $\mathrm{NPCH}$ $(2.5 \mathrm{mg} / \mathrm{kg})$ or NP $(\mathrm{CH}+\mathrm{SMB})(2.5 \mathrm{mg} / \mathrm{kg})$ and blood was collected at different time intervals, and $\mathrm{CH}$ concentration was determined by HPLC analysis. Results in Figure 7 the serum level of $\mathrm{CH}$ was almost doubled when it was administrated in $\mathrm{NP}(\mathrm{CH}+\mathrm{SMB})$ as compare to $\mathrm{CH}$ alone and $\mathrm{NPCH}$ form. It is also evident from the Figure 7 that

\section{Zeta potential of Nanocapsules}

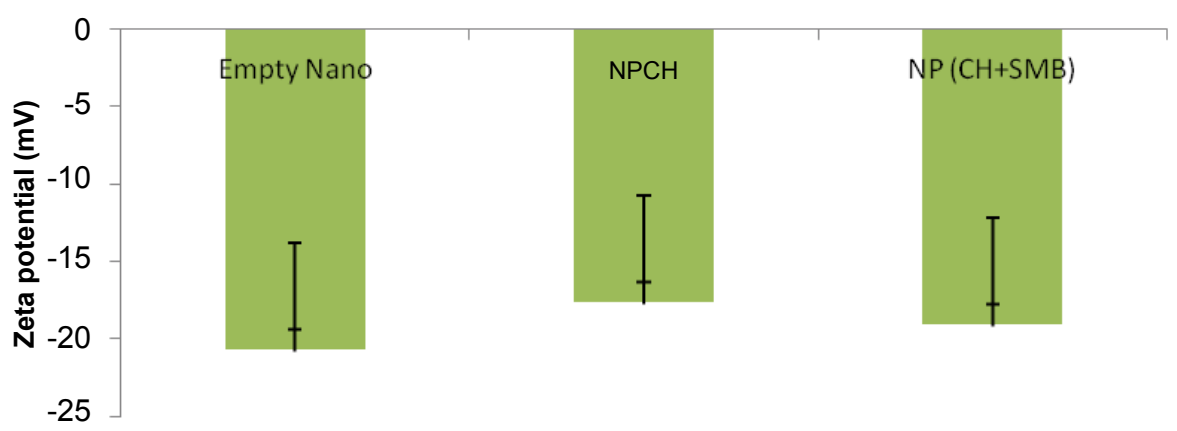

Figure 1: Stability of nanocapsules was measured as negative zeta potential within $-25 \mathrm{mV}$. Values were shown as mean $\pm \mathrm{SD}$

\section{Invitro drug release property of $\mathrm{CH}$ loaded PLGA nanocapsules}

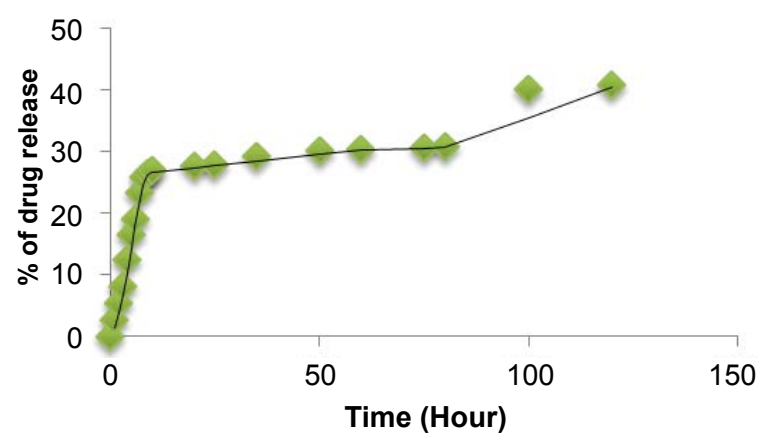

$\%$ of drug release

Log. (\% of drug release)

-2 per. Mov. Avg. (\% of drug release)

Figure 2: In vitro $\mathrm{CH}$ release pattern of $\mathrm{NPCH}$ and $\mathrm{NP}(\mathrm{CH}+\mathrm{SMB})$ with respect to different time interval was shown. 
Citation: Samanta A, Bandyopadhyay B, Das N (2016) Formulation of Catechin Hydrate Nanocapsule and Study of its Bioavailability. Med chem (Los Angeles) 6: 399-404. doi:10.4172/2161-0444.1000376
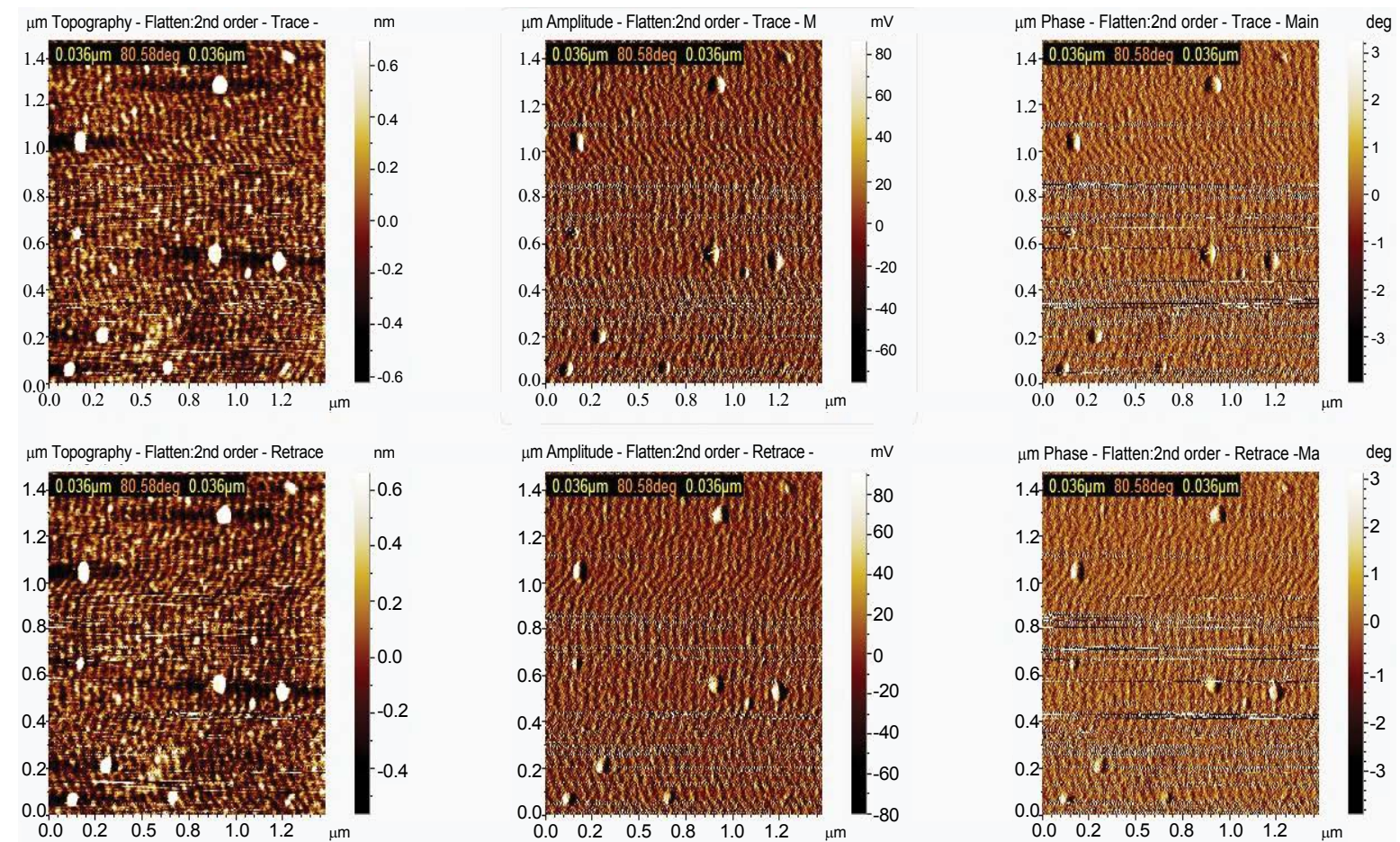

Figure 3: AFM images of drug loaded nanocapsules with their topography-flatten $2^{\text {nd }}$ order,-flatten $2^{\text {nd }}$ order and phase-flatten $2^{\text {nd }}$ order view. Horizontal cross sections indicate the height of the nanocapsules from the substratum, i.e., the mica sheet.

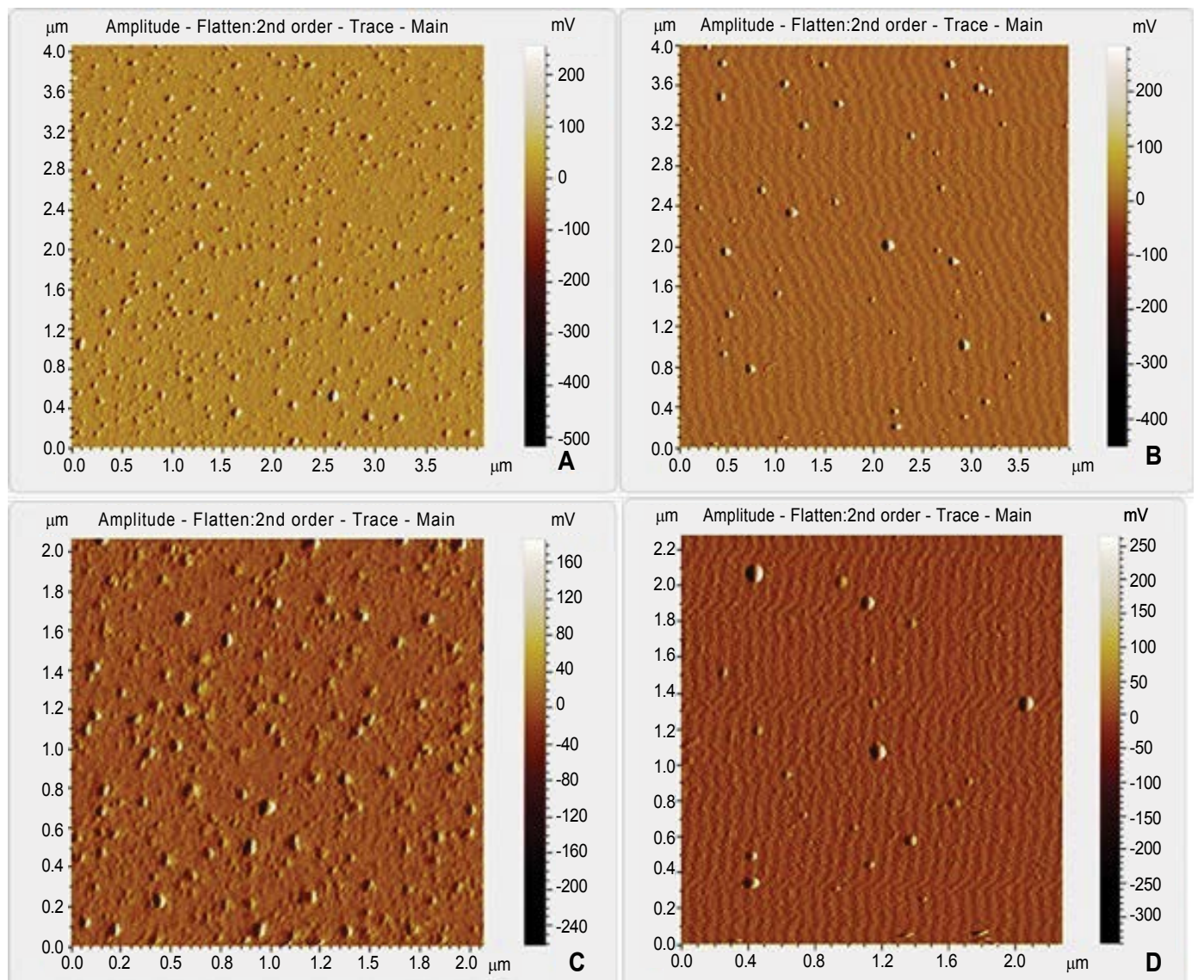

Figure 4: Individual AFM images of nanocapsules in amplitude-flatten: $2^{\text {nd }}$ order view. Images of free nanoparticle (A), NPCH (B), NPSMB (C) and NP (CH+SMB) showing spherical shape of nanocapsules. 


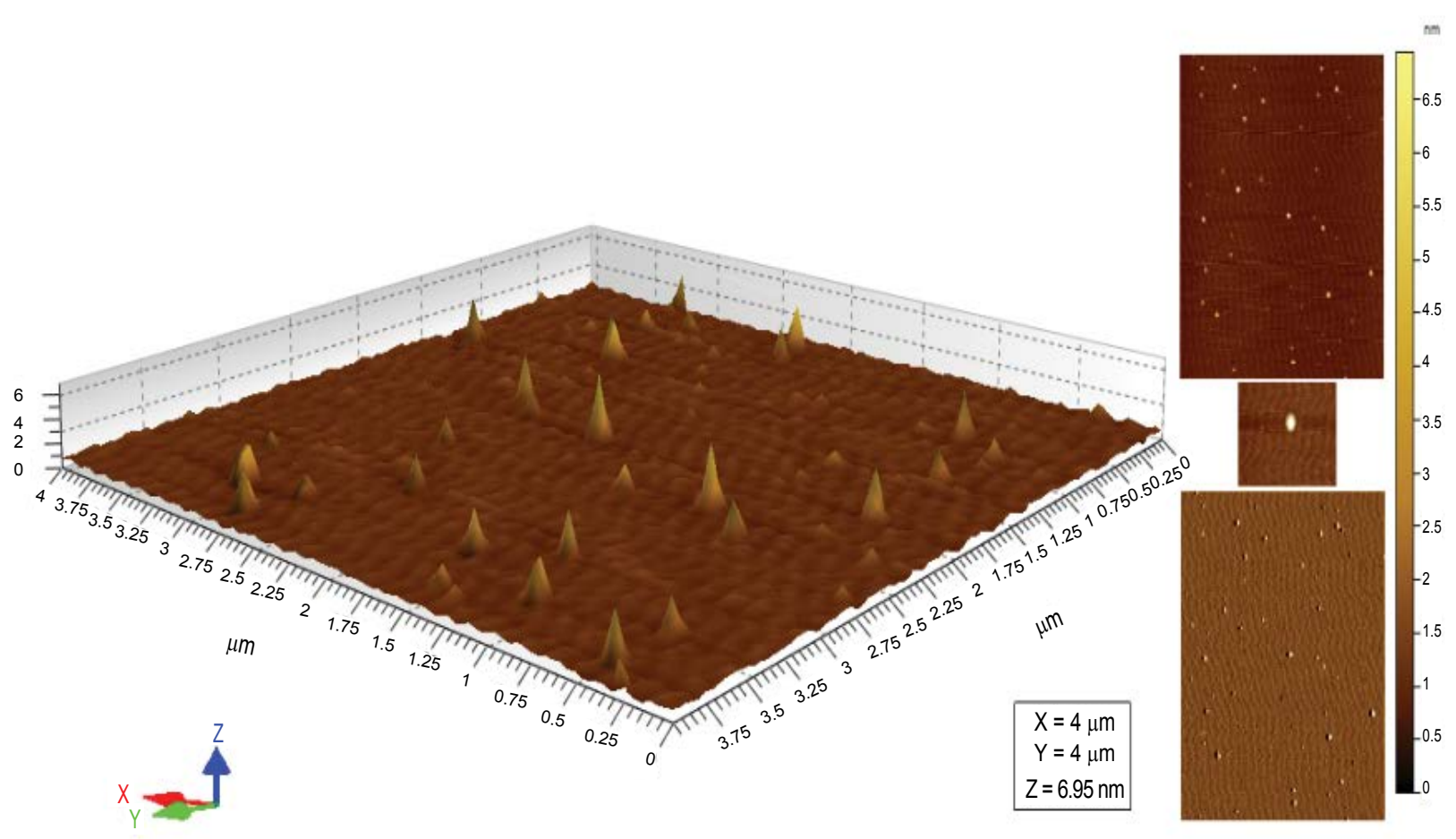

Figure 5: Three dimensional AFM image of drug loaded nanocapsules showing the mica sheet representing $6.95 \mathrm{~nm}$ height of nanoparticle. Right side of the image showing the $2^{\text {nd }}$ order topography-flatten, single and phase-flatten nanoparticles.

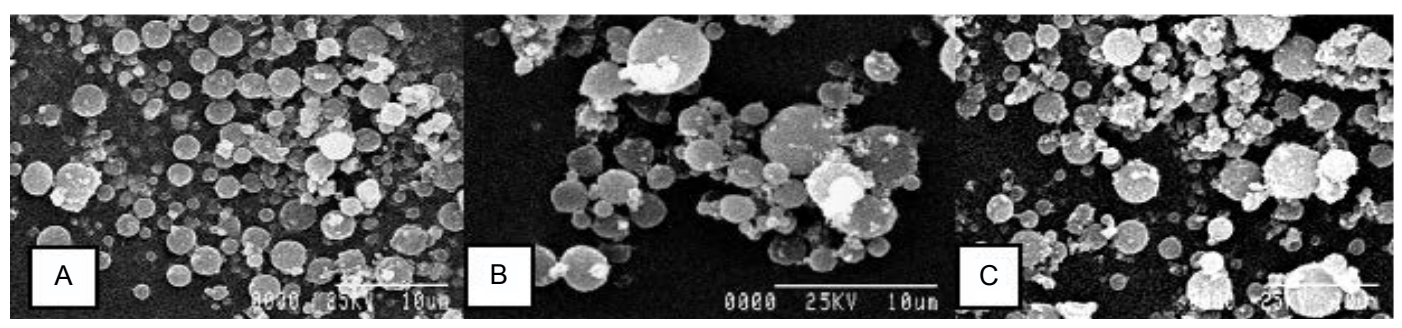

Figure 6: Scanning electron microscopy images showing drug encapsulation of NPCH (A), NPSMB (B) and NP (CH+SMB) (C).

\section{Availability of free and nanocapsulated Catechin Hydrate}

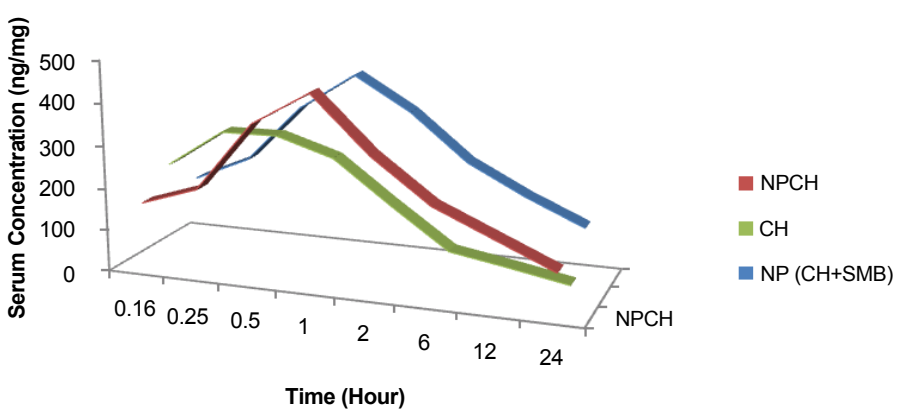

Figure 7: Bioavailability of free drug $[\mathrm{CH}]$, nanocapsulated drug $[\mathrm{NPCH}]$ and nanocapsulated drug-chelator $[\mathrm{NP}(\mathrm{CH}+\mathrm{SMB})]$. $\mathrm{Concentration}$ of $\mathrm{CH}$ and $\mathrm{NPCH}$ was determined at different time interval by collecting blood from rat groups given injection of free $\mathrm{CH}$ and $\mathrm{NPCH}$.

serum concentration of $\mathrm{CH}$ was markedly increased when it was administrated in NPCH form. In addition, half-life of NPCH and NP $(\mathrm{CH}+\mathrm{SMB})$ were substantially longer than that of free $\mathrm{CH}$. Sustained release of nanocapsulated drugs up to 3 days (Figure 2) has proved its efficacy for better bioavailability (Figure 7) and thus established its role as a suitable drug delivery vehicle. 
Cationic boranes of sodium, potassium could be used by clinicians for better management of fluoride intoxications, in combination of antioxidant drug. Our previous data suggested that nanocapsulated SMB significantly helps to chelate more fluoride as compare to free SMB due to sustainable release with a lesser dose [12]. Antioxidant drugs have the potency to neutralize the oxidative damage but dose fixing and time management is necessary to measure before its application. Our selected antioxidant drug (+)-Catechin Hydrate contains 5 hydroxyl groups, which attributes the antioxidant potency to bind directly with ROS so as to scavenge and eliminate it during oxidation by limiting lipid peroxidation. Regarding the antioxidant activity, (+)-catechin has been found to be the most powerful scavenger between different members of the different classes of flavonoids. The ability to quench singlet oxygen seems to be in relation with the chemical structure of catechin, with the presence of the catechol moiety on ring $\mathrm{B}$ and the presence of a hydroxyl group activating the double bond on ring $\mathrm{C}$ [13]. In a similar study, researchers from our laboratory have reported the synergistic effect of chelator and antioxidant drug into nanocapsule against chronic arsenic toxicity and stress induced oxidative damage [7]. The combinatorial therapy could also be effectively circumvented blood brain barrier in brain and mucosal barrier in gastrointestinal tract, leads to significant achievement in drug delivery system.

The current report discussed about the development of a biodegradable nanoparticle formulation of $\mathrm{CH}$ based on PLGA technique using DMAB as a stabilizer and tested its bioavailability and efficacy on rats where we found that nanocapsulated catechin hydrate $[\mathrm{NPCH}]$ and nanocapsulated catechin hydrate-sodium meta borate [NP $(\mathrm{CH}+\mathrm{SMB})]$ had much higher cellular uptake in vitro compared to that of free catechin hydrate. However identical cellular uptake was noticed for both the cases. This method has been used to encapsulate a wide variety of hydrophobic drugs including coenzyme Q [14], ellagic acid [15], estradiol [9], taxol [16,17], ellagic acid [15] and camptothecin [18].

Our results indicate that $\mathrm{CHNP}$ and $\mathrm{NP}(\mathrm{CH}+\mathrm{SMB})$ are likely to have great potential and could be used for further therapeutic purpose against fluoride toxicity.

\section{Conclusion}

PLGA nanoparticles were prepared and used to encapsulate, protect and release the bioactive flavonoid molecule catechin hydrate. In our study, we showed that this flavonoid was successfully encapsulated in a non crystalline state within the PLGA nanocapsule matrix using the DMAB as a stabilizer. New combinations of nanocapsule for drug delivery systems were prepared with either $\mathrm{CH}$ alone or in a combination with $\mathrm{CH}+\mathrm{SMB}$. Also we have noticed that the release profile of $\mathrm{CH}$ was sustained up to 70 hours which proved its efficacy for better bioavailability for drug delivery. These results suggest that PLGA nanocapsules with chelators such as SMB, could be suitable for encapsulation of bioactive compounds for the design and fabrication of the nanocapsule for the targeted delivery of drugs. Finally, the PLGA nano-delivery systems improve controlled release of the encapsulated $\mathrm{CH}$ flavonoids to target organ with oxidative stress. Further, novel therapeutic strategies could be investigated that might provide better therapeutic outcomes. Employing combination therapy with chelating agent and or prescribing antioxidants or nutraceuticals might be more consciously considered as crucial recommendations of chelation therapy to fluoride induced toxicity. Nevertheless, further studies are necessary to establish that PLGA nanocapsules can protect and release these flavonoids within the body system through evaluating their bio accessibility and bioavailability.

\section{References}

1. Kesarwani K, Gupta R, Mukerjee A (2013) Bioavailability enhancers of herbal origin: an overview. Asian Pac J Trop Biomed 3: 253-266.

2. Zhang L, Kosaraju SL (2007) Biopolymeric delivery system for controlled release of polyphenolic antioxidants. European polymer journal 43: 2956-66.

3. Hudnall TW, Chiu CW, Gabbaï FP (2009) Fluoride ion recognition by chelating and cationic boranes. Acc Chem Res 42: 388-397.

4. Sutherland BA, Rahman RM, Appleton I (2006) Mechanisms of action of green tea catechins, with a focus on ischemia-induced neurodegeneration. J Nutr Biochem 17: 291-306.

5. Setiawan VW, Zhang ZF, Yu GP, Lu QY, Li YL, et al. (2001) Protective effect of green tea on the risks of chronic gastritis and stomach cancer. Int $\mathrm{J}$ Cancer 92: 600-604.

6. Miura Y, Chiba T, Tomita I, Koizumi H, Miura S, et al. (2001) Tea catechins prevent the development of atherosclerosis in apoprotein E-deficient mice. Nutr 131: 27-32.

7. Ghosh S, Dungdung SR, Chowdhury ST, Mandal AK, Sarkar S, et al (2011) Encapsulation of the flavonoid quercetin with an arsenic chelator into nanocapsules enables the simultaneous delivery of hydrophobic and hydrophilic drugs with a synergistic effect against chronic arsenic accumulation and oxidative stress. Free Radic Biol Med 51: 1893-1902.

8. Lockman PR, Mumper RJ, Khan MA, Allen DD (2002) Nanoparticle technology for drug delivery across the blood-brain barrier. Drug development and industrial pharmacy 28: 1-3.

9. Hariharan S, Bhardwaj V, Bala I, Sitterberg J, Bakowsky U (2006) Design of estradiol loaded PLGA nanoparticulate formulations: a potential oral delivery system for hormone therapy. Pharmaceutical research 23: 184-95.

10. Win KY, Feng SS (2005) Effects of particle size and surface coating on cellular uptake of polymeric nanoparticles for oral delivery of anticancer drugs. Biomaterials 26: 2713-2722.

11. Oberdörster G, Elder A, Rinderknecht A (2009) Nanoparticles and the brain: cause for concern?. J Nanosci Nanotechnol 9: 4996-5007.

12. Samanta A, Chanda S, Bandyopadhyay B, Das N (2016) Establishment of drug delivery system nanocapsulated with an antioxidant $(+)$-catechin hydrate and sodium meta borate chelator against sodium fluoride induced oxidative stress in rats. J Trace Elem Med Biol 33: 54-67.

13. Tournaire C, Croux S, Maurette MT, Beck I, Hocquaux M, et al. (1993) Antioxidant activity of flavonoids: efficiency of singlet oxygen (1 delta g) quenching. J Photochem Photobiol B 19: 205-215.

14. Ankola DD, Viswanad B, Bhardwaj V, Ramarao P, Kumar MR (2007) Development of potent oral nanoparticulate formulation of coenzyme Q10 for treatment of hypertension: can the simple nutritional supplements be used as first line therapeutic agents for prophylaxis/therapy? European Journal of Pharmaceutics and Biopharmaceutics. 67: 361-369.

15. Bala I, Bhardwaj V, Hariharan S, Kharade SV, Roy N, et al. (2006) Sustained release nanoparticulate formulation containing antioxidant-ellagic acid as potential prophylaxis system for oral administration. J Drug Target 14: 27-34.

16. Kim TY, Kim DW, Chung JY, Shin SG, Kim SC, et al. (2004) Phase I and pharmacokinetic study of Genexol-PM, a cremophor-free, polymeric micelleformulated paclitaxel, in patients with advanced malignancies. Clin Cancer Res 10: 3708-3716.

17. Mu L, Feng SS (2003) A novel controlled release formulation for the anticancer drug paclitaxel (Taxol): PLGA nanoparticles containing vitamin E TPGS. Journal of controlled release 86: 33-48.

18. Vasey PA, Kaye SB, Morrison R, Twelves C, Wilson P, et al. (1999) Phase clinical and pharmacokinetic study of PK1 [N-(2-hydroxypropyl) methacrylamide copolymer doxorubicin]: first member of a new class of chemotherapeutic agents-drug-polymer conjugates. Clinical Cancer Research 5: 83-94. 